NOUVELLE

\section{NPM : un nouvel acteur dans le cancer de la prostate?}

Claude Beaudoin, Sabrina Maquaire, Laurent Léotoing, Jérôme Allemand, Corinne Lours-Calet, Georges Veyssière, Michèle Manin, Laurent Morel
$>$ Le cancer de la prostate (CaP) représente une part importante et croissante des causes de décès liés à une pathologie cancéreuse chez l'homme. Sa forte incidence dans les pays industrialisés a justifié le développement de nombreux programmes de recherche; toutefois, les mécanismes moléculaires impliqués dans la carcinogenèse prostatique restent encore mal définis. Les androgènes jouent un rôle capital dans la cancérisation de la glande prostatique et la diminution des taux circulants d'androgènes reste encore la thérapie la plus utilisée pour le traitement du CaP [1, 13]. Néanmoins, après une période de
A

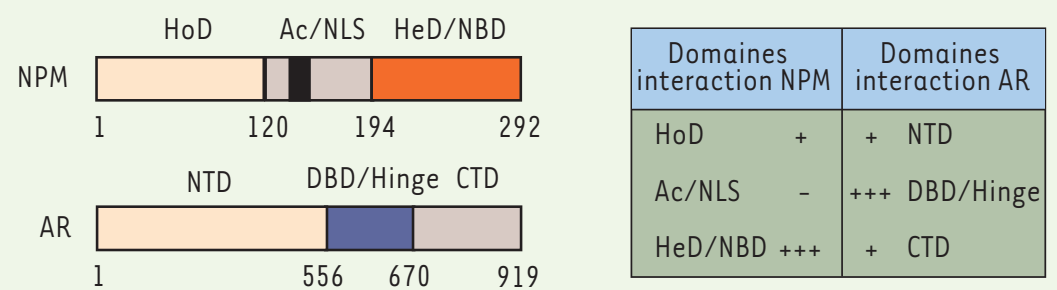

B

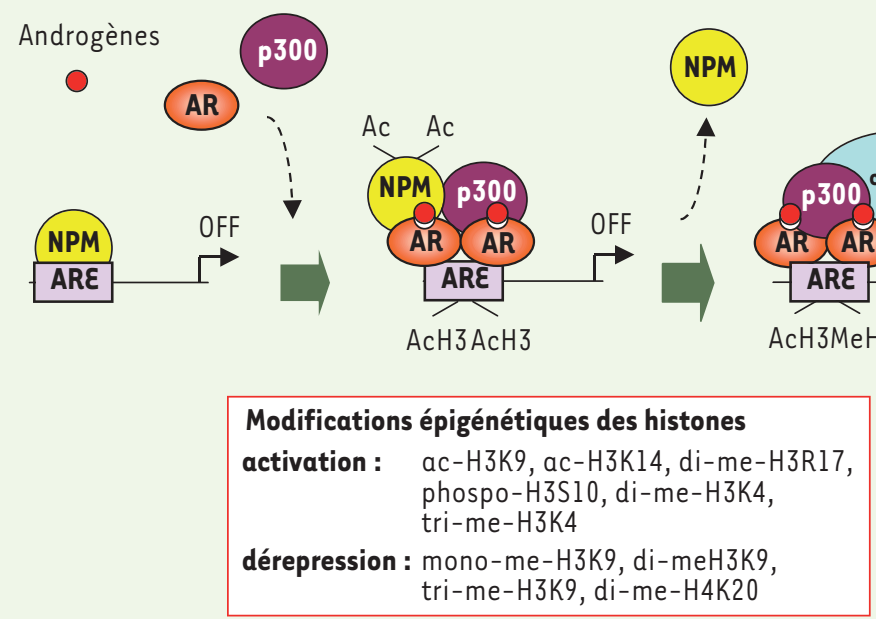

C. Beaudoin, S. Maquaire, J. Allemand,

C. Lours-Calet, G. Veyssière, M. Manin,

L. Morel : CNRS UMR 6247/

Clermont Université/INSERM U931,

Campus Universitaire des Cézeaux,

24, avenue des Landais,

63177 Aubière Cedex, France.

Laurent.MOREL@univ-bpclermont.fr

L. Léotoing : Département d'Hématologie,

Inserm U567, Institut Cochin,

24, rue du Faubourg Saint-Jacques,

75014 Paris, France.

rémission transitoire, certaines cellules prostatiques deviennent insensibles à la privation androgénique favorisant la reprise de la progression tumorale et la dissémination métastatique [2]. II semble toutefois que la signalisation androgénique soit toujours active dans les cellules qui échappent à la privation hormonale. En effet, cette phase s'accompagne d'une élévation du niveau d'expression du gène codant pour la protéine PSA (prostate specific antigen), cible de l'action des androgènes. II n'existe pas aujourd'hui de traitement

Figure 1. Domaines d'interaction et contrôle de la transcription par NPM en réponse aux androgènes. A. Le domaine amino-terminal (NTD) $\mathrm{du}$ récepteur des androgènes ( $A R$ ) renferme le domaine majeur d'activation de la transcription alors que la région centrale contient le domaine de liaison à l'ADN et la région charnière ( $\mathrm{DBD} /$ hinge). La région carboxy-terminale (CTD) contient le domaine de liaison du ligand et un domaine transactivateur mineur. NPM comprend dans sa partie amino-terminale une région hydrophobe qui permet son oligomérisation (HoD). Ce domaine est suivi par une portion centrale qui contient deux domaines acides essentiels à la liaison aux histones (Ac) NLS). La partie carboxy-terminale contient un domaine d'hétéro-dimérisation et de liaison aux acides nucléiques (HeD/NBD) [6]. B. Dans les cellules tumorales de prostate, NPM est surexprimée ce qui altère les mécanismes

régulateurs de l'activation de la transcription dépendante de AR [7]. NPM agirait comme une plate-forme de recrutement facilitant la liaison de AR et de p300 à la chromatine puis l'activation de la transcription après acétylation des histones et remodelage chromatinien (adapté de [3]). Ainsi, NPM pourrait participer à la régulation du code épigénétique des histones avec les démétylases LSD1 et JMJD2C (déméthylases contenant le domaine Jumonji (Jmj)) [11] pour activer et/ou lever la répression sur la transcription dépendante de AR et se dissocier de la chromatine après acétylation par l'acétyltransférase p300 [8] (Ac: acétylation; $\mathrm{AcH3}$ : acétylation de l'Histone H3 ; MeH3 : méthylation de l'Histone H3). 
curatif pour lutter contre le $\mathrm{CaP}$, et les questions posées concernent autant l'origine des cellules cancéreuses que les nombreuses altérations métaboliques et génétiques qui les différencient des cellules épithéliales saines [14]. Dans la cellule, les androgènes mobilisent un récepteur spécifique, le récepteur des androgènes, qui fonctionne comme un facteur de transcription inductible par son ligand. II active la transcription génique après liaison à des séquences ADN spécifiques (ARE, $A D N$ responsive element) situées dans les régions régulatrices de ses gènes cibles. À ce niveau, son action est dépendante du recrutement et/ou de son interaction avec des co-régulateurs activateurs ou répresseurs [3, 4]. On attribue donc une grande importance à la surexpression et aux mutations de ce récepteur ainsi qu'à ses protéines partenaires pour expliquer la contribution du récepteur des androgènes à la carcinogenèse prostatique et à sa phase d'échappement hormonal.

\section{NPM : partenaire du récepteur des} androgènes et oncogène prostatique? Par une approche de purification biochimique des protéines associées au récepteur des androgènes à partir de cellules épithéliales maintenues en phase de prolifération suivie de l'analyse des complexes isolés par spectrométrie de masse, nous avons identifié la nucléophosmine (NPM), une protéine multifonctionnelle impliquée dans le développement et la tumorigenèse [5, 6]. NPM est fortement exprimée avec le récepteur des androgènes dans les tumeurs prostatiques par comparaison avec le tissu sain adjacent [7]. Des études complémentaires ont ensuite démontré que NPM interagit avec le récepteur des androgènes pour contrôler l'expression de ce dernier et sa capacité à activer la transcription en réponse aux androgènes. Puisque NPM interagit fortement avec le domaine de liaison à I'ADN, nous avons également constaté que la capacité de liaison à I'ADN du récepteur est fortement augmentée lorsque le taux de NPM est plus élevé (Figure 1A) [7]. Ces résultats révèlent que NPM pourrait contrôler l'activité de transcription du récepteur des androgènes en renforçant sa capacité de liaison aux éléments cis régulateurs présents au sein des régions promotrices de ses gènes cibles. Une analyse réalisée par immunoprécipitation de chromatine (ChIP) indique que NPM se dissocie de la chromatine en présence d'androgènes alors que le récepteur est recruté de manière spécifique au niveau de ses ARE [7]. À partir de ces résultats, nous proposons que la protéine NPM facilite le recrutement du récepteur des androgènes au niveau de la chromatine pour activer la transcription en réponse aux androgènes, sans toutefois être présente dans le complexe final d'initiation de la transcription (Figure 1B).

\section{NPM et transcription dépendante} du récepteur des androgènes: la piste épigénétique.

NPM pourrait influer sur le contrôle de la transcription en régulant l'architecture de la chromatine et son accessibilité. À l'appui de cette hypothèse, il a été montré que NPM interagit avec l'histone acétyltransférase p300 pour stimuler la transcription dépendante de l'acétylation [8]. Puisque le recrutement de la protéine p300 au niveau de la chromatine est un processus essentiel à l'activation de la transcription dépendante du récepteur des androgènes [9], il apparaît probable que NPM puisse faciliter l'attachement de ce récepteur et de p300 lors de l'activation de la transcription après acétylation des histones et remodelage de la chromatine. Une surexpression de NPM pourrait avoir des conséquences importantes sur l'activation de la transcription dépendante du récepteur des androgènes dans les cellules cancéreuses de prostate en facilitant le recrutement du récepteur à la chromatine malgré de faibles taux d'hormones.

\section{Conclusion et perspectives}

Nos résultats suggèrent que NPM joue un rôle important dans le CaP en modulant l'activité transcriptionnelle du récepteur des androgènes. II est maintenant capital d'explorer la fonction oncogène de NPM dans la prostate et d'éclaircir les mécanismes régulateurs de la transcription dépendante du récepteur des androgènes. Une surexpression de NPM serait-elle suffisante pour stimuler la prolifération des cellules de l'épithélium prostatique et déclencher le développement d'un cancer chez la souris? Des mutations affectant l'expression et/ou l'activité de NPM sontelles détectables dans les cellules de CaP? Finalement, l'identification de la protéine NPM comme protéine interagissant avec le récepteur des androgènes soulève plusieurs interrogations concernant sa fonction dans le contrôle de la transcription dépendante de ce récepteur. Des analyses par ChIP fourniraient des informations décisives quant au rôle joué par NPM dans l'activation de la transcription par le récepteur des androgènes, notamment au niveau de l'acétylation des histones et du remodelage de la chromatine induits par le recrutement de $p 300$. Ces modifications pourraient participer au code épigénétique des histones déjà impliqué dans le contrôle de l'expression des gènes cibles du récepteur des androgènes [1012, 15]. Dans l'optique d'applications thérapeutiques, une meilleure connaissance du mode d'action de NPM dans la régulation de la réponse aux androgènes ouvrirait de nouvelles perspectives pour le traitement du CaP, qui est le cancer le plus fréquent de l'homme et la deuxième cause de décès par cancer. $\diamond$

NPM: a new player in prostate cancer?

\section{RéFÉRENCES}

1. Huggins C, Stevens RE, Hodges CV. Studies on prostatic cancer: II. The effects of castration on advanced carcinoma of the prostate gland. Arch Surg $1991 ; 43: 209-23$.

2. Russell PJ, Benette S, Stricker P. Growth factor involvment in the progression of prostate cancer. Clin Chem $1998 ; 44: 705-23$. 
3. Heemers HV, Tindall DJ. Androgen receptor (AR) coregulators: a diversity of functions converging on and regulating the AR transcriptional complex. Endocrinol Rev $2007 ; 28$ : 778-808.

4. Kaarbo M, Klokk TI, Saatcioglu F. Androgen signaling and its interactions with other signaling pathways in prostate cancer. Bioessays $2007 ; 29$ : 1227-38.

5. Grisendi S, Bernardi R, Rossi M, et al. Role of nucleophosmin in embryonic development and tumorigenesis. Nature 2005 ; 437 : 147-53.

6. Grisendi S, Mecucci C, Falini B, et al. Nucleophosmin and cancer. Nat Rev Cancer 2006 ; $6: 493-505$.

7. Leotoing L, Meunier L, Manin M, et al. Influence of nucleophosmin/B23 on DNA binding and transcriptional activity of the androgen receptor in prostate cancer cell. Oncogene 2008 ; $27: 2858-67$.
8. Swaminathan V, Kishore AH, Febitha KK, et al. Human histone chaperone nucleophosmin enhances acetylation-dependent chromatin transcription. Mol Cell Biol $2005 ; 25$ : 7534-45.

9. Fu M, Wang C, Wang J, et al. Androgen receptor acetylation governs trans activation and MEKKL induced apoptosis without affecting in vitro sumoylation and trans- repression function. Mol Cell Biol 2002 ; 22 : 3373-88.

10. Jia L, Shen HC, Wantroba M, et al. Locus-wide chromatin remodeling and enhanced androgen receptor-mediated transcription in recurrent prostate tumor cells. Mol Cell Biol 2006; 26 : 7331-41.

11. Wissmann M, Yin N, Muller JM, et al. Cooperative demethylation by JMJD2C and LSDI promotes androgen receptor-dependent gene expression. Nat Cell Biol $2007 ; 9$ : 347-53.
12. Metzger $\varepsilon$, Yin $N$, Wissmann $M$, et al. Phosphorylation of histone $\mathrm{H} 3$ at threonine $\mathrm{ll}$ establishes a novel chromatin mark for transcriptional regulation. Nat Cell Biol 2008; $10: 53-60$.

13. Labrie F, Cusan L, Gomez JL, et al. De la biologie à la clinique : le décès dû au cancer de la prostate peutil maintenant être une exception? Med Sci (Paris) $2003 ; 19: 910-9$

14. Cussenot 0 , Cancel-Tassin G. Facteurs de risque génétiques pour le cancer de la prostate. Med Sci (Paris) $2004 ; 20: 562-8$.

15. Ray-Gallet D, Gérard A, Polo S, Almouzni G. Variations sur le thème du «code histone ». Med Sci (Paris) $2005 ; 21: 384-9$.

\section{NOUVELLE}

\section{Régulation de la plasticité dans le cortex auditif par l'expérience sensorielle}

Étienne de Villers-Sidani, Michael M. Merzenich

\author{
W.M. Keck Center for Integrative Neuroscience, \\ Coleman Laboratory, Department of Otolaryngology, \\ University of California, San Francisco, \\ 513 Parnassus Avenue, Room HSE-808, Box 0732, \\ San Francisco, CA 94143, États-Unis. \\ etienne@phy.ucsf.edu
}

\section{Périodes critiques de plasticité durant le développement}

Dès notre naissance, le cerveau en développement est bombardé de stimulus nouveaux, propres à l'environnement unique dans lequel nous évoluons. Les circuits neuronaux du cortex immature devront alors apprendre à décoder ces signaux de manière à bâtir une représentation fidèle du monde environnant, qui conduira à des comportements adultes adaptés.

C'est à un jeune âge, lors de phases de développement bien définies - aussi appelées périodes critiques (PC), que le pouvoir plastique du cerveau est maximal. Durant ces $P C$, les réarrangements corticaux s'effectuent spontanément, sans effort, par la simple exposition à un stimulus cohérent et répétitif. À l'âge adulte, le cerveau est moins malléable et seul un entraînement intensif ou une attention soutenue peut changer de manière durable les représentations corticales, ce qui assure la stabilité des apprentissages passés [1]. Chez l'humain, le développement du langage constitue sans doute le plus fascinant exemple de plasticité corticale. Celui-ci s'effectue en étapes claires durant lesquelles des représentations mentales de plus en plus complexes sont consolidées: phonologie de 0 à 1 ans, sémantique de 1 à 4 ans, puis enfin la syntaxe et la grammaire dont la base sera maîtrisée vers 5 ans [11].

L'existence sur notre planète de centaines de langues dotées de phonologies très différentes indique clairement que cet apprentissage complexe ne peut pas être expliqué entièrement par le bagage génétique; des enfants élevés depuis un très jeune âge dans une langue différente de celle de leurs parents maîtriseront sans difficulté leur langue d'accueil. Ces périodes de grande plasticité ont cependant un prix : elles sont limitées dans le temps. Ainsi, dès l'âge d'un an, sur le plan linguistique, des nourrissons ne sont plus en mesure de distinguer des sons auxquels ils n'ont pas été exposés régulièrement, comme les sons / $r$ / et le /I/ pour les Japonais ou les Chinois, par exemple [2]. Selon les mêmes principes, une perception auditive brouillée par une perte auditive conductive modérée causera des déficits d'apprentissage de la langue à moins qu'une intervention chirurgicale corrective ne soit pratiquée avant l'âge d'un an [3].

L'étude des périodes critiques dans le cerveau a pris son essor à la suite des expérimentations historiques d'Hubel et Wiesel en 1963 sur la déprivation monoculaire chez le chaton [4] ${ }^{1}$. Depuis, l'existence des PC a été confirmée et étudiée dans plusieurs autres modalités sensitives, comme le cortex somatosensitif (SI) et le cortex auditif (Al) [5]. Dans $A l$, les fréquences sont représentées selon un axe bien défini avec les basses fréquences d'un côté et les hautes fréquences de l'autre (axe tonotopique, voir Figure l). Cette propriété d'Al,

\footnotetext{
${ }^{1}$ Dans ces expériences de Hubel et Wiesel, une paupière du chaton été maintenue fermée (cousue) pendant une période de 2-4 semaines durant le développement, bloquant ainsi toute information visuelle provenant d'un des deux yeux. À la fin de cette période, l'oeil a été ouvert, et les propriétés des neurones du cortex visuel primaire étudiées. Les auteurs ont trouvé que la majorité de ces neurones préféraient maintenant les stimulus présentés à l'oeil qui était resté ouvert, mais uniquement si l'occlusion était exécutée durant les 4-6 premières semaines de vie du chaton (période critique pour la déprivation monoculaire)
} 